Chapter 14

\title{
Reproductive Health Challenges of Living with HIV- Infection in Sub Saharan Africa
}

\author{
O. Erhabor, T.C. Adias and C.I. Akani \\ Additional information is available at the end of the chapter \\ http://dx.doi.org/10.5772/52682
}

\section{Introduction}

The human immunodeficiency Virus (HIV) pandemic is one of the most serious health crisis faced by the world today. An estimated 34 million people were living with HIV/AIDS as at 2010 [1]. A disproportionate burden has been placed on women and children who continue to experience high rates of new infection and HIV-related illness and death. Availability and use of antiretroviral drugs has changed the landscape of HIV/AIDS bringing about a change in the perception of HIV from an incurable deadly disease to a chronic manageable illness. As effective HIV treatments become more widespread, HIV-infected individuals in sub Saharan Africa are living longer, healthier lives. Many HIV-affected couples (sero-discordant and sero-concordant) are now considering long-term life projects including options for safer reproduction or procreation. There has also been increase in advocacy to expand the capacity for the health care system particularly in Africa to provide the sexual and reproductive health services that HIV infected persons in Africa desperately need [2]. This decade has witnessed greater commitment to sexual and reproductive health and HIV linkages particularly in the developed world. More recent opportunities include policy developments within the Global Fund to Fight AIDS, Tuberculosis and Malaria to accept proposals that form linkages with sexual and reproductive health within the overall frameworks of HIV, tuberculosis (TB) and malaria. There has also been a renewed commitment by the United States of America to international sexual and reproductive health through support of the United Nations Population Fund and the repeal of the Mexico City Policy, also known as the "global gag rule", a United States government policy that hitherto prohibited non-governmental organizations from receiving federal funding for performing or promoting abortion services in other countries. A large body of evidence suggests that reproductive technologies can help HIV-affected couples to safely conceive with minimal risk of HIV transmission to their part- 
ner and baby. However, for most couples particularly in low income countries in sub Saharan Africa, such technologies are neither geographically nor economically accessible. In subSaharan Africa where HIV is endemic, 63\% of women have an unmet need for sexual and reproductive health services, there is a high incidence of unintended pregnancies, a significant number of women do not know their HIV status, many women have limited access to sexual and reproductive health information and services (family planning, management of sexually transmitted diseases, HIV prevention and maternal health) to help them protect themselves from the triad of unwanted pregnancy, HIV infection and HIV transmission to their sexual partners and their children. With HIV now considered to be a chronic manageable disease, attention is shifting to offering and improving quality of life particularly by the provision of reproductive health options/care to men and women living with HIV-1.

A healthcare workforce that is highly motivated and well informed on the evidence -based practices in sexual and reproductive health options for persons living with HIV/AIDS is crucial to meeting the sexual and reproductive needs of PLWHA in Africa. A high morale and productive workforce are the driving force for the success of any reproductive health programme. Unless these staffs are motivated and have high morale, they may become a stumbling block to the success and scaling -up of sexual and reproductive health services [3]. There are several challenges associated with meeting the sexual and reproductive health needs of persons living with HIV/AIDS in Africa; suboptimal antenatal care, absence of evidence-based and affordable assisted reproductive technologies, inadequate number of appropriately trained healthcare workers, suboptimal health infrastructure, lack of enabling legislation and policies on sexual and reproductive health of HIV-infected persons, challenge of stigmatization and discrimination, unprofessional negative attitudes towards PLWHA desiring to procreate. The aim of this chapter is to highlight the reproductive health concerns associated with living with HIV infection in sub Saharan Africa.

\section{History of Africa}

Africa is the world's second-largest and second-most-populous continent in the planet, after Asia. With 1,032,532,874 billion people as at 2011 [4]. It accounts for about $14.72 \%$ of the world's human population. At about 30.2 million $\mathrm{km}^{2}$ (11.7 million sq miles) including adjacent islands, it covers $6 \%$ of the Earths' total surface area and $20.4 \%$ of the total land area [5]. It is made up of 54 member states. Western Sahara although a member of the African union, its sovereignty is being disputed by Morocco. South Sudan has become Africa newest nation having recently separated from North Sudan. The population of Africa is estimated at greater than one billion people. Africa account for a significant $14 \%$ of the world's population. Africa contains the Nile River system which is the worlds longest. It also prides itself as the continent with the world's largest Sahara desert. Africa is surrounded by the Mediterranean Sea (north) and the Suez Canal and the Red sea (northwest), the Indian Ocean (east) and the Atlantic Ocean (west). Although endowed with abundant natural resources, Africa remains the world's poorest and most underdeveloped continent, the result of a variety of causes that may include the spread of deadly 
viruses and diseases (HIV/AIDS, malaria and tuberculosis), corruption in government that have often committed serious human right abuses and violations, failed central planning, high levels of illiteracy, lack of access to foreign capital, and frequent tribal and military conflict (ranging from guerrilla warfare to genocide). According to the United Nation's Human Development Report in 2003, the bottom 25 ranked nations (151st to 175th) were all African [6].Poverty, illiteracy, malnutrition and inadequate water supply and sanitation, as well as poor health, affect a large proportion of the people who reside in the African continent. About $80.5 \%$ of the Sub-Saharan Africa population lives on less than $\$ 2.50$ per person per day in 2005 [7]. Africa faces several daunting challenges with regards to access to basic health services like their counterparts in most developed countries of the world. The healthcare system and infrastructure are suboptimal. This is often due to fundamental limitations in funding, lack of adequate qualified health professional and equipment as well as deep rooted, institutionalized and chronic corruption among the political class and bureaucratic compensation and corruption among civil servants [8]. Africa remains the world's most corrupt continent. Corruption is the abuse of entrusted power for private gain, in public and private sectors [9]. This vice has contributed to a large extent to the stunted development and impoverishment seen in many African states. The African union estimates that corruption among the political class is costing the continent more than $\$ 150$ billion dollars per year. These are funds that could be used to improve the health infrastructure and quality of life of people of people in the continent but rather are laundered out of developing countries to banks in the developed world thus perpetrating poverty among African people. Industrialized countries have continued to encourage corruption in Africa and perpetuation of poverty among people in the African continent by providing crooked African leaders with a safe haven for their looted funds rather than repatriating such funds back and ensuring that they are used to enhance the infrastructural development of the continent. Corruption is endemic and continues to thrive in the African continent for several reasons; institutional weakness and criminal collaboration between the executive and the legislative and judicial arms of government, non-existence of the principle of rule of law, political god fatherism, institutional failure and criminal collaboration between civil servants and politicians. Corruption is a cankerworm that continues to weaken societies, ruins lives, and impedes development in the African continent [10]. This is further compounded by the high incidence of infectious diseases (HIV, TB and Malaria). Africa is plagued by poverty, malnutrition, poor sanitation, disease, high mortality rate, conflict, wars and crime. These challenges have a significant negative effect on life expectance in the continent. The continent has the world's shortest life expectancy. Citizens of Sub-Saharan African countries are much more likely to die prematurely, than people in wealthier parts of the world. Children under- the age of 5 years are more likely to die from malaria, respiratory tract infections, diarrhoea, perinatal conditions, measles and HIV/AIDS while those who survive the first 5 years of life are likely to die before their $60^{\text {th }}$ birthday from HIV/AIDS, tuberculosis, and maternal mortality (for women as a result of pregnancy-related mortality). According to the CIA World Fact book 2009 [11], the life expectancy at birth of the world is 67.2 years (65.0 years for males and 69.5 years for females). The United Nations World Population Pros- 
pects 2006 Revision put the world's life expectancy at birth at 66.57 years (64.52 years for males and 68.76 years for females).Women on the average are found to live longer than men with the exception of Zimbabwe, Afghanistan, Swaziland and Lesotho [12].Countries in Africa particularly those with a high HIV/AIDS prevalence have must lower life expectancies [11]. Provision of low technology, safer, affordable and readily available reproductive health options particularly for PLWHA in Africa is a crucial but often unaddressed component of HIV prevention programme. The aim of this chapter is to evaluate the sexual and reproductive health challenges associated with HIV infection in sub Saharan Africa.

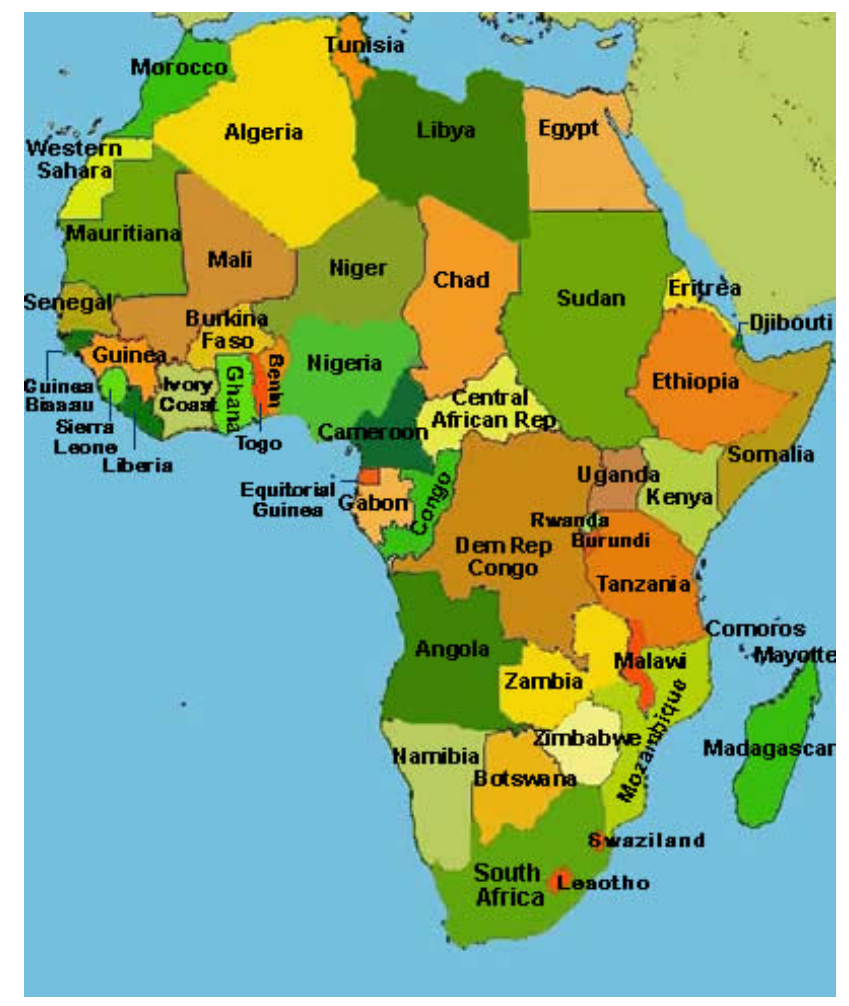

Figure 1.

\section{Challenge of suboptimal antenatal services in Africa}

Organized preventive screening programmes for antenatal care were first introduced in Western Europe in the twentieth century with the hope that routine antenatal care would contribute to a reduction in maternal and infant mortality rates. Figures on maternal mortal- 
ity in the developed world show that the risk of death as a result of pregnancy and child birth is approximately 1 in 7000 compared to 1 in 23 for women living in many parts of Africa where antenatal care is poor or non-existent [13]. Antenatal care is an opportunity to reach the mothers and young girls in a safe non-stigmatising environment. The importance of developing links between sexual and reproductive health and HIV services is widely recognized. Four priority areas for linkages has been identified- learning about one's HIV status, promoting safer sex, optimizing links between HIV and sexually transmitted infection services and integrating HIV with maternal and infant health. These priorities could lead to significant public health benefits and improve efficient use of resources particularly in resource -constrained settings in Africa. There are many challenges and issues that affect reproductive health choices particularly for persons living with HIV in Africa. They include; paucity of evidenced-based information regarding safe pregnancy and Prevention of Mother To Child Transmission (PMTCT) of HIV, absence of universal access to Voluntary Counselling and Testing (VCT) services, negative attitudes by society and un-informed healthcare workers towards PLWHA desiring to have children, lack of universal access to condoms, contraceptives medications and Highly Active Antiretroviral Therapy (HAART), sup-optimal levels of skilled sexual and reproductive health staffs, lack of service infrastructures and absence of legislation on the reproductive health right of PLWHA. All these factors negatively affects and complicates the ability of PLWHA to make evidenced based reproductive health choices particularly in many sub Saharan African settings.

Antenatal care creates an opportunity for women to access HIV testing and counselling. It is the port of entry to accessing HIV prevention and care. In 2001, CDC modified the recommendations for pregnant women to emphasize HIV screening as a routine part of prenatal care, simplification of the testing process so that pre-test counselling would not pose a barrier, and flexibility of the consent process to allow multiple types of informed consent [14].Many pregnant women particularly in Africa do not know their HIV serostatus. Many particularly those in rural settings lack adequate information on the benefit of antenatal care for themselves and their babies. High level of illiteracy, inequalities in access to healthcare services, being pregnant at an early age, women dependence economically on men, limited mobility, poverty, religious and cultural restrictions, being a member of a marginalized community or population remains major barriers or stumbling blocks that militate against access to effective antenatal particularly in resource-constrained settings in sub Saharan Africa [15]. Women all over the world will most likely accept VCT services if it is offered especially in settings where there is universal access to HAART and evidenced-based effective sexual and reproductive services rendered by staff who are appropriately trained to render the best possible evidenced-based counselling about HIV and other STIs as well as affordable and readily available reproductive health options with empathy rather than being judgemental. Humiliating and stigmatising attitudes and breaches of confidentiality of antenatal women can create a barrier that can potentially prevent women from accessing sexual and reproductive health services even when they are available.

There are several ways to possibly enhance the uptake of VCT services in Africa. They include approaches such as the "opt-out" approach, use of traditional voluntary counselling 
and testing strategies as well as making antenatal visit as interactive and activity based. These approaches are more likely to provide the much needed coverage [16]. There has been argument as to whether the "opt-out" approach negates the principle of informed consent [17] particularly in African settings where many antenatal women are reluctant to challenge health care procedures. It is important however to note that "opt-out" approach does not in any way compromise the principle of informed consent. This approach assumes that testing is an intrinsic part of an effective and holistic antenatal care. The women are informed about what test is required as well as the importance of testing to the mother and the developing baby with an opportunity given to women to refuse testing if they so desire.

There is also the need to foster more male involvement in antenatal care in Africa. Evidence has shown that involving men in the reproductive health care of their partners rather than basing antenatal care exclusively on women can potentially enhance the pregnancy experience, reduces incidence of gender-based violence, promote the likelihood of getting a joint consent, facilitate HIV prevention, compliance to HAART in partner in which it is indicated as well as enables a holistic uptake of sexual and reproductive health service for couples. There are several challenges that militate against male-gender involvement in antenatal care particularly in Africa; cultural and religious beliefs, obstacles of working fathers, prevalence of polygamy and maintenance by men of multiple sex partners [18-19].

When HIV infection is diagnosed, health-care providers should strongly encourage patients to disclose their HIV status to their spouses, current sex partners, and previous sex partners and recommend that these partners be tested for HIV infection. Health departments can assist patients by notifying, counselling, and providing HIV testing for partners without disclosing the patient's identity [20]. Policy allowing providers to inform patients who receive a new diagnosis of HIV infection that they might be contacted by health department staff for a voluntary interview to discuss notification of their partners may play a beneficial role and facilitate uptake of sexual and reproductive health services in Africa. A disproportionate burden has been placed on women and children who continue to experience high rate of new HIV infection and HIV-related illness and death. Most children living with HIV acquire the infection through Mother to Child Transmission (MTCT) which can occur during pregnancy, during delivery or during breastfeeding.

\subsection{A strong cultural and religious attachment to having children puts PLWHA under pressure to procreate}

Being HIV positive has been shown to modify but not remove the reproductive desires of individuals. Diversity existed in reproductive intentions among PLWHA. Some HIV positive individuals wished to avoid pregnancy. Fears of partner and infant infection and having a previously infected baby were important factors deterring some individuals from considering having children. There is also strongly perceived community disapproval associated with HIV and reproduction. Strong desires to experience parenthood, mediated by prevailing social and cultural norms that encouraged childbearing in society have also been reported. Motherhood is seen as an important component of married women's identity and important for women's social status in Africa. Family, husbands and societal expectations 
for childbearing have significant influences on the African woman's reproductive intentions [21]. Availability and use of antiretroviral drugs has changed the landscape of HIV/AIDS bringing about a change in the perception of HIV from an incurable deadly disease to a chronic manageable illness. As effective HIV treatments become more widespread, HIV-infected individuals are living longer, healthier lives. Many HIV-affected couples (sero-discordant and sero-concordant) are considering options for safer reproduction. A large body of evidence suggests that reproductive technologies can help HIV-affected couples to safely conceive with minimal risk of HIV transmission to their partner and baby. However, for most couples particularly in low income countries in sub Saharan Africa, such technologies are neither geographically nor economically accessible. With HIV now considered to be a chronic manageable disease, attention is shifting to offering and improving quality of life particularly by the provision of reproductive health options/care to men and women living with HIV-1. Many HIV-infected men and women are now expressing their desire to father or mother a child. Assisted reproductive technologies, including intrauterine insemination (IUI), in vitro fertilisation (IVF) and intracytoplasmatic sperm injection (ICSI) in combination with semen washing have been used to decrease the risk of HIV -1 transmission in HIV-1infected discordant couples with an HIV-1-infected man. Previous report indicates that in HIV-positive men taking HAART, seminal viral load is decreased but not eliminated and fertilization should be achieved through sperm washing to offer maximum protection for the uninfected female. Pregnant HIV-positive women on antiretroviral medication have a reduced risk of transmitting the virus, but should still be counselled about the possibility to further limit the chances of infecting their infant through elective Caesarean section. HIV sero-discordant couples with strong desire for childbearing have a dilemma of risking HIV infection or infecting their spouse. Some risk transmission of HIV infection to reproduce. Over two-thirds of 104 surveyed couple wanting to procreate reported unprotected sex with their partner in the past 6 months. Most respondents, regardless of serostatus, said that viral load testing and awareness of post-exposure prevention had no effect on their condom use. A paucity of interventions targeting sero-discordant couples on contraceptive choices is at odds with a strong cultural importance in Africa attached to having children. HIV discordance in Africa creates a serious dilemma for fertility decision-making in couples. Stigma, discrimination, and non-disclosure fuel HIV transmission between partners. A previous study [22] in Nigeria a country in the Western part of Africa that investigated the reproductive health concerns among persons living with HIV/AIDS in the Niger Delta of Nigeria has shown that a significant number of PLWHA in Nigeria have conception dreams. The main reasons for wanting to procreate included: ensuring lineage continuity and posterity (52.3\%), securing relationships $(27.0 \%)$ and pressure from relatives to reproduce $(20.7 \%)$. Single subjects were more inclined to have children $(76.3 \%)$ compared to married $(51.5 \%)$, widowed $(18.2 \%)$ and separated/divorced $(11.1 \%)(\mathrm{p}=0.03)$. Of the 111 subjects that indicated their desire to have children, women were more inclined to have children (64.5\%) compared to men $(47.7 \%)$. The major concern among the $84(43.1 \%)$ subjects not desiring more children were the fear of infecting sero-discordant partner and baby (57.1\%), fear of dying and leaving behind orphans $(28.6 \%)$ and the fear that they may become too ill and unable to financially support the child (14.3\%). Persons with no formal education were more likely to have 
children irrespective of their positive HIV status (66.7\%) compared to persons educated to tertiary education level $(37.0 \%)(\mathrm{p}=0.01)$. Out of the 111 subjects that desired to have more children, only $58 \%$ had gone for reproductive health counselling with HIV counsellors. Reasons for not seeking advice were anticipated negative reactions and discrimination from the counsellors. A significant number of subjects were only aware of some reproductive health options available to reduce risk of infecting their partners and or baby such as artificial vaginal insemination, intrauterine insemination, caesarean section, avoidance of breast feeding and offering prenatal pre-exposure prophylaxis to the foetus. They were unaware of other options such as sperm washing, IVF and ICSI. Of the $43.1 \%$ not anticipating more children, $36.9 \%$ were anticipating adoption.

A significant number of PLWHA in Africa desire to have children irrespective of the HIV positive status. Women are more inclined to have children compared to men. Persons with no formal education are more inclined to have children irrespective of their positive HIV status compared to persons educated to tertiary education level. The main reasons for wanting a child included: ensuring lineage continuity and posterity, securing relationships and pressure from relatives to reproduce. There may be several reasons for this association, including the fact that better educated people generally having greater access to information particularly the mode of transmission of HIV than those who have less formal education, and are more likely to make informed decisions and act on information given. In addition, better educated people generally have better jobs and greater access to money and other resources which can help them lead healthier lives. Also single persons living with HIV are more likely to want to have children compared to married, separated and widowed subjects. Regardless of interpersonal and public health concerns, studies in both resource-rich and resource-limited settings suggest that HIV-infected men and women desire children [23 - 25]. In addition, in resource limited settings, couples often desire larger families. The reasons for this are debated but likely include among others, the strong cultural attachment to having children, stigmatization associated with childlessness, role of children with inheritance, the importance of children in agricultural economies, the importance of childbearing on the status of women, the role of children as caretakers of the elderly, and high rates of infant mortality [26]. Previous report indicated that forty percent of HIV infected women desired more children and women with fewer children were more likely to become pregnant [27].

PLWHA are often not wanting to procreate because of the fear of infecting sero-discordant partner and baby, fear of dying and living behind orphans and the fear that they may become too ill and unable to financially support the child.Previous report indicates that the major challenges faced by HIV-infected subjects not desiring to procreate included: risk of HIV transmission to partner and child and failure of health systems to offer safe methods of reproduction [28]. Identifying the determinants of the decision to have children among serodiscordant couples will help in setting reproductive intervention priorities in resource-poor countries. The gender of the positive partner affects the factors associated with a desire for children. Interventions targeting sero-discordant couples should explore contraceptive choices, the cultural importance of children, and partner communication [29]. 
Given the importance of procreation in African settings and the lack of low -technology and affordable assisted reproduction services, HIV-infected couples are faced with a serious dilemma about making an informed decision to procreate. Many PLWHA in Africa who have a desire to have children do not seek reproductive health counselling from HIV counsellors. Reasons for not seeking counselling were anticipated negative reactions and stigmatization from the counsellors resulting from their negative attitude towards unprotected sexual activity and child bearing by HIV-infected couples. HIV-infected individuals and their partners are requiring education and counselling regarding HIV disease and reproduction and HIV counsellors particularly in Africa do not seem to have access to evidenced -based information that the HIVinfected population desperately need to enable them make informed reproductive health decisions. Previous report suggest that there is need to draw up a protocol for reproductive counselling of HIV infected that have a desire for conception [30]. HIV counsellors need to come to terms with the fact that simply encouraging HIV- infected couples to abstain from procreation may no longer be a realistic strategy, particularly in sub Saharan Africa where there is strong cultural attachment to having children. In the absence of counselling that recognizes the desire and importance of having children, couples may knowingly take on the risks of transmission in order to have children. Sharing our evidenced-based best practices about HIV transmission and reproductive health options while recognizing patient goals may help couples minimize risk and reduce the harm of unprotected sex. However, the great risks taken by HIV -infected persons desiring to procreate could be minimized through counselling and close monitoring by reproductive health care provider.

Reproductive health knowledge among HIV-infected subjects desiring to procreate in Africa is poor. Most PLWHA in Africa are unaware of reproductive health options such as sperm washing, in vitro fertilization (IVF) and intracytoplasmic sperm injection (ICSI) available to reduce risk of infecting their partners and or baby. A significant number of men taking HAART have lower seminal concentration of HIV, and sexual transmission may be reduced. However, a certain percentage of aviraemic men retain viral presence in semen, and unprotected intercourse to achieve fertilization must be discouraged as it carries the risk of sexual transmission of the virus. HIV-discordant couples should be informed that sperm washing can remove HIV from semen, allowing conception without the risk of infection for the seronegative female and eventually the child. In HIV-positive women, perinatal transmission of HIV can be curtailed to less than $2 \%$ by using HAART to decrease maternal viral load and offering prenatal pre-exposure prophylaxis of the foetus, and elective Caesarean section. Each intervention carries specific risks and benefits. The contribution of each preventive arm in achieving foetal protection can only be crudely measured and optimal obstetric management must involve discussion with the pregnant woman of the pros and cons of each strategy. HIV-affected couples who want to have children is presented with at least three distinct and daunting clinical challenges. The first is dealing with stigma arising from many health care providers' negative attitude towards sexual activity and child bearing by HIV infected couples [31] and stigmatization from immediate family members and society [32]. The second is maintaining the mother's health before, during and after pregnancy. The third is preventing vertical transmission from mother to child as well as preventing HIV transmission to the partner in sero-discordant relationship. Several approaches have been suggested to re- 
duce risk of horizontal transmission for HIV-affected couples who want to conceive children. These approaches includes the use of ; male sperm washing, IUI, ICSI, screening and pre-treatment for Sexually Transmitted Infections (STI's), Delay in procreation until viral load is controlled, limited, timed unprotected sexual encounters, female artificial insemination, self-insemination and circumcision. Experience among couples in whom the male was (HIV) seropositive who underwent assisted reproductive technologies (ART) in order to attain family goals while minimizing the risk of HIV transmission indicated that all female recipients tested seronegative for HIV at 3 and 6 months post-embryo transfer. All delivered babies $(n=8)$ tested seronegative for HIV at birth and 3 months postpartum and that ART should be considered for HIV serodiscordant couples who desire to have children in order to minimize the risk of viral infection [33]. Several people play a role in reproductive health decision making of PLWHA; the relatives who used traditional norms to encourage procreation; the health workers who violates the autonomy and human rights of HIV-infected by using their medical knowledge to dissuade clients from childbearing by preaching mandatory contraception [34] and the health care system that do not recognize and meet the sexual and reproductive health needs of their clients [35]. Health care providers in Africa must realize that it is their responsibility to offer information to enable HIV-infected persons arrive at their own informed decisions on their reproductive and sexual health needs regardless of the health professional's opinion. Similarly, there may be need to offer additional training to enable counsellors' particularly in sub Saharan Africa offer evidenced- based sexual and reproductive health information to their clients. Reproductive health policies in this HIV/AIDS era are lacking in most African settings. It is recommended that sero-discordant couples who desire to have children should undergo assisted fertility treatment such as sperm washing, intra uterine insemination and in-vitro fertilization to avoid HIV transmission to their partners [36]. However cost implication is a major issue affecting the feasibility of offering assisted fertility treatment such as sperm washing, intra uterine insemination and in-vitro fertilization particularly among low socioeconomic people. There is a major challenge with the development of evidenced- based, cost- effective and best-practice guidelines in most settings in Africa to optimize the sexual and reproductive health service rendered to persons living with HIV/AIDS. In resource-limited settings, couples should be counselled on ovulation cycles and may engage in timed unprotected sex only during the fertile period of the woman's monthly cycle to facilitate conception while reducing number of exposures. If the man is HIV-negative with a positive partner, partners can be taught artificial insemination, timed to the woman's fertile period. For couples in which both partners are positive, there may be need for a careful and informed natural conception when their viral loads have fallen to below the level of detection.

\section{Lack of integration between sexual and reproductive, family planning and existing HIV /AIDS services}

There is increasing concern that Sub-Saharan Africa is the region where more women are infected by HIV than men. About 60 per cent of people living with HIV infections in Afri- 
ca are women. Among young men and women aged 15 to 24 years, for every one man, four women are infected with HIV. There were 12-13 infected women to 10 infected men in 2001 [37]. Biological, cultural and socio-economic factors contribute to women's greater vulnerability to HIV/AIDS. Women are 4 times more at risk of becoming infected with HIV during unprotected vaginal intercourse than men. The vagina's greater area of susceptible tissue and micro trauma that occurs during sexual intercourse makes women more physiologically more vulnerable [38]. Semen has higher viral load than vaginal fluids and the semen stays longer in the female genital tract after sexual intercourse which increases the chances of HIV transmission. The synergy between HIV and sexually transmitted infection (STIs) is another biological factor that makes women more vulnerable to HIV. This is especially significant among African women. Most STI cases in women often go untreated, symptoms are often latent, women diagnosed with STIs are often stigmatised and majority have no access to medical treatments [39]. Socio-economic factors including women's lack of access to education, personal income, and economic dependence on men perpetuate women's lower status. Moreover widespread poverty often drives these vulnerable women into commercial sex work. Furthermore men control over condom use- the main tool for reducing the risk of sexual transmission of HIV puts women at risk of HIV. Cultural practices inherent in Africa such as forced marriage, polygamy, female genital mutilation and older men's preferences for sex with younger women, Sexual violence coercion at home and in the work place during job hiring, promotion and to avoid dismissal are common practices, use of women as bait by companies to secure contracts, sexual abuse of orphans and domestic workers further complicates female gender vulnerability to HIV [40]. Moreover, women are more subjected to HIV stigma and discrimination. In Africa, the HIV virus that causes AIDS is transmitted through two major routes. The first, which accounts for 80 per cent of the cases, is through unprotected sex between men and women. This is followed by HIV transmission from mother to child during pregnancy, labour and breastfeeding, which is responsible for about 20 per cent of the cases. There are several biological, social and cultural reasons why women and girls in Africa are more vulnerable to HIV infection. Gender inequalities that exist in African society, women have less access to information than men, they are less likely to make informed decisions and act on information given, they have less access to education, and better jobs, money and other resources which can help them lead healthier lives. During vaginal sex, which is commonly practiced in Africa, the chance of HIV transmission from a man to a woman is two to three times greater than transmission from a woman to a man. This is due to the biological make up of the female genital tract. The female genital tract has a larger area of exposed tissue. Young girls are especially vulnerable when they have sex with older men because because the genital tract of young girls are immature, prone to tear and invasion by HIV.

There has never been a better time than this to target and integrate sexual and reproductive health, family planning and HIV/AIDS services particularly in Africa. Most sceptics erroneously often consider reproductive health to be a euphemism for abortion services. It is worthy to note that reproductive health covers a broad range of women's health issues including detecting and treating sexually transmitted infections and supporting HIV-infected women's desire to have children safely. Integration of HIV and reproductive health has 
the potential to produce important HIV-related outcomes. Recent international consensus statements have urged the strengthening of these linkages [41]. In order to reduce HIV-infected births, infant and child mortality, the number of children orphaned by AIDS and maternal mortality, adding family planning and reproductive health to PMTCT, VCT and ARV programmes makes a logical and a programmatic sense.

\section{HIV-infected need information and services on safe procreation and the health system is unable to provide evidenced-based answers}

Data from most settings in Africa increasingly demonstrate that some HIV-infected women particularly those on ARVs as they begin to feel better and function more normally due to the effect of treatment on reduction on viral load, would like to become pregnant [42]. It is a growing expectation that the health system should have the ability to counsel HIV-positive women on the risk and benefits of child bearing and to respect the reproductive intentions, choices and rights including access to contraception and other reproductive health services. Previous report [22] indicates that reproductive health knowledge among HIV-infected subjects desiring to procreate was poor. Subjects were unaware of reproductive health options such as sperm washing, in vitro fertilization (IVF) and intracytoplasmic sperm injection (ICSI) available to reduce risk of infecting their partners and or baby. A significant number of men taking HAART have lower seminal concentration of HIV, and sexual transmission may be reduced. However, a certain percentage of aviraemic men retain viral presence in semen, and unprotected intercourse to achieve fertilization must be discouraged as it carries the risk of sexual transmission of the virus. HIV-discordant couples should be informed that sperm washing can remove HIV from semen, allowing conception without the risk of infection for the seronegative female and eventually the child. In HIV-positive women, perinatal transmission of HIV can be curtailed to less than $2 \%$ by using HAART to decrease maternal viral load and offering prenatal pre-exposure prophylaxis of the foetus, and elective Caesarean section. Each intervention carries specific risks and benefits. The contribution of each preventive arm in achieving foetal protection can only be crudely measured and optimal obstetric management must involve discussion with the pregnant woman of the pros and cons of each strategy. Several approaches have been suggested to reduce risk of horizontal transmission for HIV-affected couples who want to conceive children. These approaches includes the use of ; male sperm washing, IUI, ICSI, screening and pre-treatment for Sexually Transmitted Infections (STI's), delay in procreation until viral load is controlled, limited, timed unprotected sexual encounters, female artificial insemination, self-insemination and circumcision. Accurate and accessible information to make informed choices and safe, pleasurable sexual relationships possible is best delivered through peer education and health professionals trained on empathetic approaches to sensitive issues [43]. Interventions based on positive prevention, which combine protection of personal health with avoiding HIV/STI transmission to partners, are recommended. 


\section{Non-protection of the reproductive rights of PLWHA in Africa}

It is increasing clear particularly that a significant majority of PLWHA in Africa are of reproductive age, that conception and reproductive options for this population are important issues for health care delivery and research and that HIV-seropositive individuals deserve full reproductive rights like every other person [44]. International reproductive guidelines shifted a decade ago from recommending avoidance of pregnancy to recognizing conception and parenting as realistic options and rights for people with HIV infection and their partners [45]. Mindful of this indisputable fact, US Centers for Disease Control and Prevention (CDC) has encouraged information and support for HIV-affected couples who want to explore their reproductive options [14]. There are many persons who plays a role in reproductive health decision making of persons living with HIV/AIDS: The relatives who used traditional norms to encourage procreation; the health workers who violates the autonomy and human rights of HIV-infected by using their medical knowledge to dissuade clients from childbearing by preaching mandatory contraception [34] and health care system that does not recognize and meet the sexual and reproductive health needs of their clients [35].Health care providers in Africa must realize that it is their responsibility to offer information to enable HIV-infected persons arrive at their own informed decisions on their reproductive and sexual health needs regardless of the health professional's opinion. Similarly, there may need to offer additional training to enable counsellors' offer evidenced- based sexual and reproductive health information to their clients. Reproductive health policies in this HIV/AIDS era are lacking in most African settings. It is recommended that sero-discordant couples who desire to have children should undergo assisted fertility treatment such as sperm washing, intra uterine insemination and in-vitro fertilization to avoid HIV transmission to their partners [36, 46]. However cost implication is a major issue affecting the feasibility of offering assisted fertility treatment such as sperm washing, intra uterine insemination and in-vitro fertilization particularly among low socioeconomic people. There is a major challenge with the development of evidenced- based, cost effective and best-practice guidelines locally in most African setting to help optimize the sexual and reproductive health service rendered to persons living with HIV/AIDS particularly in the Africa. Like it is in other developed countries in the world. HIV-infected couples wanting to procreate in Africa should be counselled on ovulation cycles, timed sex only during the fertile period of the woman's monthly cycle to facilitate conception while reducing number of exposures. If the man is HIVnegative with a positive partner, partners can be taught artificial insemination, timed to the woman's fertile period. For couples in which both partners are positive, there may be need for a careful and informed natural conception when their viral loads have fallen to below the level of detection. There is the need to support the sexual and reproductive rights of HIV-infected individuals. Additional training needs to be offered to HIV counsellors on evidence- based best and affordable practices regarding reproductive health issues among persons living with HIV. Policies that support the availability and accessibility to relevant reproductive and sexual health services including contraception and procreation needs to be developed. Public enlightenment programmes on HIV is needed to reduce the stigmatization that HIV-infected persons face from family members and their communities. Developing and testing safer conception methods that reduce HIV transmission to HIV-seronegative partners in serodiscordant couples and reduce 
superinfection in HIV-seroconcordant couples is a crucial component of HIV prevention programme that needs to be urgently addressed in Africa [47].

\section{Challenge of HIV-related stigma and discrimination}

HIV-related stigma and discrimination remains an enormous barrier to the fight against AIDS. Fear of discrimination often prevents people from getting tested, seeking treatment and admitting their HIV status publicly. Since laws and policies alone cannot reverse the stigma that surrounds HIV infection, AIDS education in Africa needs to be scaled-up to combat the ignorance that causes people to discriminate. The fear and prejudice that lies at the core of HIV and AIDS discrimination needs to be tackled at both community and national levels. There is strong ethical imperative to support the sexual and reproductive health needs of HIV-infected individuals allowing them to make informed decisions about their reproductive health. Increasingly, fertility clinics in developed countries are offering their services to HIV-serodiscordant couples where the woman is seropositive and in HIV-seroconcordant relationships. Reproductive health care workers in Africa can learn from the evidenced- based best practices in the developed world to ensure that like their counterparts in most developed countries, HIV infected persons particularly in Africa can access the best quality reproductive and sexual health service. Recent advances in HIV clinical care and assisted reproduction technique (ART) procedures directed at reducing the risk of viral transmission during gamete transfer particularly where good healthcare is available has significantly reduced the risk of transmission of HIV among discordant couples to 1-2\%. Promotion of risk reduction counselling, screening for sexually transmitted diseases and lower genital tract disease, assessment of options for birth control, and pre-conception counselling should be integral components of gynaecologic health care for HIV-infected women.

Since the beginning of the human immunodeficiency virus (HIV) epidemic, stigma and discrimination (SAD) have been identified as the major obstacles to effective responses to HIV [48]. HIV-affected couples who desire sexual and reproductive health services are faced with at least three distinct and daunting challenges. The first is dealing with stigma arising from many health care providers' negative attitude towards sexual activity and child bearing by HIV infected couples [49] and stigmatization from immediate family members and society [32]. The second is maintaining the mother's health before, during and after pregnancy. The third is preventing vertical transmission from mother to child as well as preventing HIV transmission to the partner in sero-discordant relationship. HIV/AIDS-related SAD has been extensively documented among health care providers. There have been many reports from health care settings of HIV testing without consent, breaches of confidentiality, labelling, gossip, verbal harassment, differential treatment, and even denial of treatment. HIV-infected-individuals who feel stigmatized by health care providers face problems accessing HIV testing and other sexual and reproductive health care services [50]. The fear of stigma impedes prevention efforts, including discussions of safer sex and the prevention of mother-to-child transmission [51]. 
Sexually transmitted infection including HIV have always been imbued with stigma and discrimination particularly in Africa particularly due to their negative association with behaviour considered by society as deviant or immoral [52]. Stigma generally refers to negatively perceived defining characteristics either tangible or intangible. It is an attribute used to set the infected person or group from the normalized social order. It has a way of devaluing a person [53]. Similarly; societies have historically reacted with fear to disfiguring, debilitating, and fatal diseases and have translated this aversion into discriminatory actions against the infected [54]. The HIV/AIDS pandemic has presented the world with a condition that combines these characteristics - and it has frequently been met with stigma and discrimination, a reaction dubbed "the second epidemic" [55]. HIV infection affects women and men's view of parenthood. It has a negative impact on their ability to have children, related not only to psychosocial aspects such as stigma and discrimination and decreased sexual activity, but also to the clinical impact of HIV infection and sexually transmitted infections (STIs) on fertility [56-57]. Learning more about stigma is important given the growing assertions that testing is a 'critical gateway' to HIV prevention and treatment. As access to HIV testing and treatment improves, providers increasingly need to understand and address how stigma acts as a barrier to services. There is need to develop programs to address the negative service provider attitudes towards HIV-positive women, especially those wanting children. Stigma and discrimination is a population and health system level barrier that discourages HIV-infected women and men from seeking reproductive health counselling and other sexual and reproductive health counselling.

HIV-related stigmatization, discrimination and denial continue to characterize the pandemic in Africa and present a major challenge to the effectiveness of prevention, sexual and reproductive care and treatment programmes. Much of the societal and individual reaction towards people with HIV/AIDS is stigma and discrimination oriented. Stigmatization and discrimination occurs in a variety of forms. It ranges from societal level responses such as coercive government policies and laws, to apathy and denial of the HIV epidemic. At the individual level, the internalization of these societal responses may result in an individual's self-exclusion from information, treatment and care. Stigmatization and discrimination are often explored through socio-cultural understandings of illness and disease transmission and its manifestations at societal and individual level. Contexts of discrimination include employment, health care systems, and travel and migration restrictions. Although there are widespread reports of HIV-related discrimination throughout the world there has also been significant progress towards reducing these practices. In addition to what is being done there is still much that we need to understand about the forms and contexts of stigmatization and discrimination if we are to succeed in our efforts to control the HIV epidemic particularly in Africa [58]. Stigma and discrimination in Africa can be challenged. One way to reduce their impact is at the legislative level, it is also vital to focus on community-based interventions. These projects target stigmatization manifested in a wide range of community contexts, including: family and immediate community, workplace, health services, religion and the media. 


\section{Need for the development and implementation of affordable, readily available, low technology and safer conception services}

Many HIV-infected men and women are now expressing their desire to have children. Although no conception methods are 100\% risk -free of HIV infection other than those that use fresh sperm from an HIV negative donor or adoption. The strong desire among Africans to have their own biological children makes these options untenable for many PLWHA. However there is several risk reduction method for safer conception in which the HIV infected partner is on antiretroviral therapy to achieve a significant reduction of viral load and increase in CD4 count. Assisted reproductive technologies, including intrauterine insemination (IUI), in vitro fertilisation (IVF) and intracytoplasmatic sperm injection (ICSI) in combination with semen washing have been used to decrease the risk of HIV -1 transmission in HIV-1-infected discordant couples with an HIV-1-infected man particularly in developed countries. Previous report indicates that in HIV-positive men taking HAART, seminal viral load is decreased to undetectable limits but not eliminated and fertilization should be achieved through sperm washing to offer maximum protection for the uninfected female. Pregnant HIV-positive women on antiretroviral medication have a reduced risk of transmitting the virus, but should still be counselled about the possibility to further limit the chances of infecting their infant through elective Caesarean section. HIV sero-discordant couples with strong desire for childbearing have a dilemma of risking HIV infection or infecting their spouse. Some risk transmission of HIV infection to reproduce. Majority of HIV -infected couples wanting to procreate are having unprotected sex with their partners. Most persons living with HIV infection need information on viral load testing, awareness of postexposure prevention and condom use. A paucity of interventions targeting HIV serodiscordant couples on contraceptive choices is at odds with a strong cultural importance in Africa attached to having children. HIV discordance creates a serious dilemma for fertility decision-making in couples. However, majority of these risk reduction method for safer conception are either unavailable regionally in Africa or unaffordable. Other low-technology and readily available and affordable option that could be used in Africa and other resource- constrained settings include; timed unprotected sexual intercourse for HIV concordant couples, vaginal insemination of fresh semen into the vagina via a disposable pipette or syringe, for HIV positive men with sero negative partners, the use of antiretroviral drugs by the HIV-infected male partner to lower HIV viral load in seminal plasma to undetectable levels, use of pre-exposure prophylaxis (PrEP) by the HIV sero-negative female partner prior to and following timed unprotected sex at the female partners ovulation period. It is vital that couples practice safer sex practices after conception and throughout the pregnancy. Previous report [59] has shown that HIV horizontal seroconversion occurred in couples who reported unsafe sex practices during pregnancy after conception. American researchers and clinicians have advocated the possibilities of using PrEP for safer conception [60]. A Recent study has indicated the potential role of PrEP in the prevention of heterosexual HIV transmission [61]. Similarly, the Pre-exposure Prophylaxis Initiative (IPrEx) trial using tenofovir/ emtricitabine has shown that PrEP is safe and capable of producing a reduction in HIV infection risk in HIV-seronegative men who have sex with men [62]. Al- 
so the CAPRISA 004 trial showed that the use of $1 \%$ tenofovir topical gel reduced the rate of HIV acquisition by 39\% in heterosexual seronegative women [63]. The use of PrEP is rapidly growing as an important component of safer conception programs for HIV-serodiscordant couples [47, 64]. In the face of the high HIV prevalence in most African setting, it is expedient that low -technology, safer conception methods that is feasible, affordable and are acceptable is implemented.

\subsection{Sperm washing with intrauterine insemination}

Several studies have reported on the efficacy of sperm washing in combination with IUI in terms of pregnancy rates, live birth rates, and HIV transmission incidence [65- 67]. However, evaluation of the efficacy of this safer conception strategy is limited by methodological issues, including small sample sizes, lack of standardized protocols, and non-rigorous study designs. For example, most studies reported only on retrospective data and very few used control groups. Sperm washing and insemination lower transmission risk for HIV-negative women who want to have children with HIV-positive men. Data from the European experience which included a 14 years of follow up for 1,036 sero-discordant couples with an HIVpositive male resulted in 580 pregnancies and no HIV sero conversions [68].

Intracytoplasmic sperm injection (ICSI).ICSI is a high-technology in vitro fertilization procedure in which a single sperm is injected into an egg. It is a popular assisted reproduction technique used as a safe conception technique in HIV seropositive men and their partners. Previous reports have shown that ICSI is particularly useful and effective in the prevention of HIV among serodiscordant couples in which the man is HIV-seropositive [46, 69]. However there are a number of challenges associated with its utility particularly in Africa. These include high cost, increased risk of multiple pregnancies and the risk of use of an HIV-infected gamete [68]. It is crucial to introduce harm-reduction methods and safer conception methods for people with HIV infection in settings where assisted reproductive technologies are unavailable and unaffordable. However, these strategies are not feasible on a widespread basis in resource-constrained settings. Sperm washing and ICSI is not feasible in most resource-constrained settings. Its high cost, the invasive nature of the procedure, the high number of cancelled cycles, increased risk of multiple pregnancies and potential danger of using an HIV-infected gamete all militate against the use of ICSI. The most practicable method in most settings in Africa is vaginal insemination with an uninfected male partner's sperm during the fertile time of the woman's menstrual cycle. Several studies in Africa have evaluated the outcome of natural concention in HIV sero-discordant couples. Ryder and colleagues [70] studied 178 married HIV sero-discordant couples in the Democratic Republic of Congo between 1987 to 1990 . Couples engaged in unprotected sex during the moment ovulation period and resulted in the birth of 24 children and one sero -conversion. Similarly a cross sectional study [71] involving 55 HIV-positive Nigeria women on antiretroviral therapy married to HIV-sero-negative men indicated that younger women compared to older women were more likely to pursue natural conception options than assisted reproduction. 


\section{Factors to consider before implementation of safer conception in sub Saharan Africa}

National medical societies such as the American Society of Reproductive Medicine and the American College of Obstetricians /Gynaecologist has re-affirmed that it is unethical to refuse to provide safer conception services to PLWHA [72].The protection of the sexual and reproductive health of all people including PLWHA has been recognized as a fundamental human right. PLWHA particularly in Africa have the right to choose to have children and to access non-judgemental, non-stigmatised and non-discriminatory evidenced-based quality sexual and reproductive services. There are however factors that need to be considered in implementing safer conception intervention; the feasibility, availability and affordability of the intervention, the need for antiretroviral therapy to ensure a low viral load, high CD4 count, absence of AIDS defining symptoms and STIs, the need for couple anticipating conception to be in a stable relationship [73], consideration of fertility problems in HIV-infected population, concomitant low success rate with assisted reproductive technologies in PLWHA, low pregnancy rate and high fetal death rates among HIV-infected women [74].

\section{Conclusion}

Sustained and increased investment in sexual and reproductive health services in Africa promises tremendous benefits to women, families and societies. In addition to improved health, sexual and reproductive health services contribute to economic growth, societal and gender equity, and democratic governance. Evidence has shown that a significant number of HIV-infected persons in Africa desire to have children irrespective of their positive sero status. There is the need to support the sexual and reproductive rights of HIV-infected individuals. Additional training needs to be offered to HIV counsellors on evidenced- based best and affordable practices regarding reproductive health issues among persons living with HIV. There is the urgent need to develop policies that support the availability and accessibility to relevant reproductive and sexual health services including contraception and procreation. There is also the need for public enlightenment programmes on HIV to reduce the stigmatization that HIV-infected persons in sub Saharan Africa face from family members and their communities. Implementation of explicit policies recognizing reproductive rights and choice of HIV-infected in Africa as well as support for health counselling and service interventions that advance safer and healthier reproductive options for HIV positive individuals is advocated. Intrauterine insemination (IUI) and Intracytoplasmic sperm injection ICSI does not seem a cost-effective option in Africa and other resource-constrained settings. However vaginal insemination with the sperm of an HIV-seronegative male partner is highly feasible and has been found to be reasonably acceptable to both men and women and reduces the risk of transmission of HIV from infected women to their uninfected partners. This may be a practicable, affordable, low- technology, safer conception option that may need to be implemented in Africa and other resource-limited settings. There is increasing advocacy that timed, limited, unprotected sex for HIV-seroconcordant couples, and timed, 
unprotected sex accompanied by periconceptionPrEP for the HIV seronegative female partner in serodisconcordant relationship could form part of a harm-reduction strategy to reduce exposure to HIV when planning conception in resource-limited settings. There is the need to increase the awareness, understanding, and acceptability of readily available, affordable, evidenced-based, low-technology and safer conception strategies among PLWHA in Africa. Evidenced based -best practices needs to be implemented in Africa to avoid HIV transmission enabling HIV-affected couples to embark on safer childbearing and to prevent the risk of mother-to-child HIV transmission as well as infection of the uninfected partners particularly in sero-discordance relationships. Countries in Africa must now come to terms that failure of the health system to engage HIV-seropositive women and men in fertility management and denying safer conception services to PLWHA who want to conceive a child is unethical and a deprivation of their fundamental reproductive right.

\section{Author details}

O. Erhabor ${ }^{1 *}$, T.C. Adias ${ }^{2}$ and C.I. Akani ${ }^{3}$

*Address all correspondence to: n_osaro@yahoo.com

1 Department of Medical Laboratory Science, Usmanu Danfodio University Sokoto, Nigeria

2 Bayelsa State College of Health Technology, Bayelsa State, Nigeria

3 Department of Obstetrics and Gynaecology, University of Port Harcourt Teaching Hospital, Nigeria

\section{References}

[1] UNAIDS (2011). Report of the global AIDS epidemic. November 2011.

[2] Stratchan, M., Kwateng-Addo, A., Hardel, K., et al. (2004). An analysis of family planning content in HIV/AIDS, VCT and PMTCT policies in 16 countries. Policy Working Paper Series, Washington DC Policy Project, 2004.(9)

[3] Kober, K,van., \& Damme, W. (2004). Access to antiretroviral treatment in Southern Africa: WHO will do the job? Lancet; , 364(982), 103-107.

[4] Sayre AP. Africa, Twenty-First Century Books. (1999). 0-76131-367-2.

[5] Richard Sandbrook (1985). The Politics of Africa's Economic Stagnation. Cambridge University Press, Cambridge, 1985 passim.

[6] The developing world is poorer than we thought, but no less successful in the fight against poverty.World Bank. 
[7] Couch JF, Atkinson KE, Shughart WF (1992). Ethics Laws and the Outside Earnings of Politicians: The Case of Alabama's 'Legislator-Educators'” Public Choice. , 73(2), 135-45.

[8] Transparency International, The anti-corruption catalyst: realising the MDGs by (2015). Transparency International, and www.transparency.org.

[9] Transparency International. Corruption PerceptionsIndex, TransparencyInternational, www.transparency.org.

[10] CIA World Fact book 2009.

[11] CIA-The World Fact book (2008). Rank order HIV/AIDS-Adult prevalence rate.

[12] United Nations. (2006). revision of the United Nations World Population Prospects report, for. , 2005-2010.

[13] Carroli, G., Rooney, C., \& Villar, J. (2001). How effective is antenatal care in preventing maternal mortality and serious morbidity? An overview of the evidence. PaediatrPerinatEpidemiol ; , 15(1), 1-42.

[14] CDC ((2001). ). Revised recommendations for HIV screening of pregnant women. MMWR; 50 ( ):63-85.(RR-19)

[15] Almeida SD, Barros MB (2005). Equity and access to health care for pregnant women in Campinas (SP), Brazil. RevistaPanamericana de SaludPublica; , 17(1), 15-25.

[16] O', Connor. K. E., \& Mac, Donald. S. E. (2002). Aiming for zero: preventing motherto-child transmission of HIV. Canadian Medical Association Journal; , 166(7), 909-910.

[17] IPAS (2005). Reproductive rights for women affected by HIV/AIDS.

[18] Mullick, S., Kunene, B., \& Wanjiru, M. Involving men in maternity care: health service delivery issues. Agenda: Special focus on Gender, Culture and Rights (Special (124-135), 124-135.

[19] UNAIDS/02.31E (2002). Keeping the promise. Summary of the Declaration of Commitment on HIV/AIDS. United Nations General Assembly Special Session on HIV/ AIDS (UNGASS), June 2001.Geneva: UNAIDS., 25-27.

[20] CDC (1998). HIV partner counselling and referral services guidance. Atlanta, GA: US Department of Health and Human Services, CDC.

[21] Cooper, D., Harries, J., Myer, L., Orner, P., \& Bracken, H. (2007). Life is still going on. Reproductive intentions among HIV-positive women and men in South Africa. Social Science and Medicine; , 65(2), 274-278.

[22] Erhabor, O., Akani, C. I., \& Eyindah, . (2012). Reproductive health options among HIV-infected persons in the low-income Niger Delta of Nigeria. HIV AIDS (Auckl); , 4, 29-35. 
[23] Frodsham, L. C., Boag, F., Barton, S., \& Gilling-Smith, C. (2006). Human immunodeficiency virus infection and fertility care in the United Kingdom: Demand and supply. Fertility and Sterility, 285 EOF-289 EOF.

[24] Myer, L., Morroni, C., \& Rebe, K. (2007). Prevalence and determinants of fertility intentions of HIV-infected women and men receiving antiretroviral therapy in South Africa. AIDS Patient Care and STDs, 278 EOF-285 EOF.

[25] Matthews LT, Mukherjee JS (2009). Strategies for harm reduction among HIV-affected couples who want to conceive. AIDS Behav; , 1, 5-11.

[26] Sonko SI. (1994). Fertility and culture in Sub-Saharan Africa: A review. International Social Science Journal.

[27] Allen, S., Meinzen-Derr, J., Kautzman, M., Zulu, I., Trask, S., Fideli, U., et al. (2003). Sexual behaviour of HIV discordant couples after HIV counselling and testin. g. AIDS

[28] Beyeza-Kashesya, J., Kaharuza, F., Mirembe, F., Neema, S., Ekstrom, A. M., \& Kulane, A. 2 EOF-12 EOF.

[29] Beyeza-Kashesya, J., Ekstrom, A. M., Kaharuza, F., Mirembe, F., Neema, S., \& Kulane, A. (2010). My partner wants a child: a cross-sectional study of the determinants of the desire for children among mutually disclosed sero-discordant couples receiving care in Uganda. BMC Public Health; 13, 10, 247.

[30] Barreiro, P., Duerr, A., Beckerman, K., \& Soriano, V. (2006). Reproductive options for HIV-serodiscordant couples. AIDS Rev; 8(3), , 158 EOF-70 EOF.

[31] de Bruyn, M. (2004). Living with HIV: Challenges in reproductive health care in South Africa. African Journal of Reproductive Health; , 8(1), 92-98.

[32] Kalichman SC, Simbayi LC (2003). HIV testing attitudes, AIDS stigma and voluntary HIV counselling and testing in a black township in Cape Town, South Africa. Sex TransmInfec; , 79, 42-447.

[33] Peña, J. E., Klein, J., Thornton, M. H., \& 2nd, Sauer. M. V. (2003). Providing assisted reproductive care to male haemophiliacs infected with human immunodeficiency virus: preliminary experience. Haemophilia, 9(3), 309-316.

[34] Stern AM (2005). Sterilized in the name of public health: race, immigration and reproductive control in modern California. Am J Public Health, 2005; , 95, 1128-1138.

[35] Sauer M V (2003). Providing fertility care to those with HIV: Time to re-examine healthcare policy. The American Journal of Bioethics , 3(1), 33-40.

[36] Semprini, A. E., Levi-Setti, P., Bozzo, M., et al. (1992). Insemination of HIV-negative women with processed semen of HIV-positive partners. Lancet, 340, 1317-1319.

[37] Geeta RG (2002). How men's power over women fuel the HIV epidemics.Br Med J; , 324(7331), 183-184. 
[38] Peter, K. L., Kristen, R., Willard, C., (2002, , \& , H. I. V. A. I. D. (2002). HIV/AIDS evolving impact on global health. In: Ron Valdisiern (ed). Dawning answers: How the HIV/AIDS epidemic has strengthened public health. New York. Oxford press.

[39] UNAIDS (1997). Women and AIDS. UNAIDS point of view. UNAIDS. Geneva.

[40] Gomez AC, Marin VB (1996). Gender, culture and power: Barriers to HIV-prevent. ion strategies for women. Journal of Sex Research; , 33(4), 355-362.

[41] The Glion Call to Action on family planning and HIV/AIDS in women and children" a consensus advocacy statement from a consultation in May 2004.

[42] Coopera, D., et al. (2005). Reproductive intentions and choices among HIV-infected individuals in Cape Town, South Africa. Lessons for reproductive policy and service provision from a qualitative study", Policy Brief, Women's Health Research Unit and Infectious Diseases epidemiology Unit, University of Cape Town, South Africa and the population council, New York, September 2005.

[43] Shapiro, K., \& Ray, S. (2007). Sexual health for people living with HIV. Reproductive Health Matter; , 15(29), 67-92.

[44] Mantell JE, Smit JA, Stein ZA (2009). The right to choose parenthood among HIV-infected women and men. J Public Health Policy. ; , 30, 367-378.

[45] Fakoya, A., Lamba, H., Mackie, N., et al. (2008). British HIV Association, BASHH and FSRH guidelines for the management of the sexual and reproductive health of people li. ving with HIV infection 2008. HIV Med; , 9, 681-720.

[46] Ohi, J. P. M., Wittemer, C., et al. (2003). Assisted reproduction techniques for HIV serodiscordant couples: 18 months of experience. Hum Reprod; , 18, 1244-1249.

[47] Chadwick, R. J., Mantell, J. E., Moodley, J., Harries, J., Zweigenthal, V., \& Cooper, D. (2011). Safer conception interventions for HIV-affected couples: implications for resource-constrained settings. Top AntivirMed ; , 19(4), 148-55.

[48] Nyblade, L., \& Mac, Quarrie. K. (2006). Can We Measure HIV/AIDS-related Stigma and Discrimination? Current Knowledge about Quantifying Stigma in Developing Countries. Washington, DC: United States Agency for International Development.

[49] Shelton JD, Peterson EA. (2004). The imperative for family planning in ART therapy in Africa. Lancet, 364, 1916-1918.

[50] Sayles, J. N., Wong, Kinsler. J. J., Martins, D., \& Cunningham, W. E. (2009). The association of stigma and self-reported access to medical care and antiretroviral therapy adherence in persons living with HIV/AIDS. J Gen Intern Med; , 24(10), 1101-1108.

[51] Obermeyer, C. M., \& Osborn, M. (2007). The utilization of testing and counselling for HIV: a review of the social and behavioural evidence. Am J Public Health; ,97(10), 1762-1774. 
[52] Goldin, C. (1994). Stigmatization and AIDS: Critical issues in public health." Social Science and Medicine; , 39(9), 1359-1366.

[53] Norbert, G., \& Somerville, M. (1994). Stigmatization, scapegoating and discrimination in sexually transmitted diseases: Overcoming them and us. Social Science and Medicine; , 39(3), 1359-1366.

[54] Angelo, A., \& Reynolds, N. (1995). Stigma, HIV and AIDS: An exploration and elaboration of a stigma trajectory." Social Science and Medicine; , 41(3), 303-315.

[55] Somerville, Margaret and Andrew Orkin ((1989). ). "Human rights, discrimination and AIDS: Concepts and issues."AIDS; 3(1): S -S287., 283.

[56] Zaba, B., \& Gregson, S. (1998). Measuring the impact of HIV on fertility in Africa..HIV-related stigma and discrimination have been shown to impede prevention, care and treatment. AIDS; , 12(1), 341-350.

[57] Paiva, V., Filipe, E. V., Santos, N., Lima, T. N. ., \& Segurado, A. (2003). The right to love: The desire for parenthood among men living with HIV. Reproductive Health Matters, 11(22), 91-100.

[58] Malcolm, A., Aggleton, P., Bronfman, M., Galvão, J., Mane, P., \& Verrall, J. (1998). HIV-related stigmatization and discrimination: Its forms and contexts.Critical Public Health; , 8(4), 347-370.

[59] Mandelbrot, L., Heard, I., Henrion, E., \& Henrion, R. (1997). Natural conception in HIV-negative women with HIV-infected partners.Lancet . 13, 2314-2315.

[60] Lampe MA, Smith DK, Anderson GJ, Edwards AE, Nesheim SR (2011). Achieving safe conception in HIV-discordant couples: the potential role of oral pre-exposure prophylaxis (PrEP) in the United States. Am J Obstet Gynecol.204: 488.

[61] Baeten, J., Celum, C., et al. (2011). The partners PrEP Study Team. Antiretroviral preexposure prophylaxis for HIV-1 prevention among heterosexual African men and women: the Partners PrPE Study(Abstract MOAX01) $6^{\text {th }}$ IAS Conference on HIV Pathogenesis, Treatment and Prevention. July Rome Italy., 17-20.

[62] Grant RM, Lama JR, Anderson PL, et al (2011). Pre-exposure chemoprophylaxis for HIV prevention in men who have sex with men. N Engl J Med; , 363, 2587-2599.

[63] Abdool, Karim. Q., Abdool, Karim. S. S., Frohlich, J. A., et al. (2010). Effectiveness and safety of tenofovir gel, an antiretroviral microbicide, for the prevention of HIV infection in women. Science; 329: 1168.

[64] Mathews, L. T., Baeten, J. M., Celum, C., \& Bangsberg, D. R. (2010). Periconception pre-exposure prophylaxis to prevent HIV transmission: benefits, risks and challenges to implementation.AIDS; , 24, 1975-1982.

[65] Semiprini AE (2000). Viral transmission in ART risks for patients and healthcare providers. Hum Reprod; 15:69. 
[66] Manigart, Y., Rozenberg, S., Barlow, P., Ge-rard, M., Bartrand, E., \& Delvigne, A. (2006). ART out-come in HIV-infected patients. Hum Reprod; , 21, 2935-2940.

[67] Savasi, V., Ferrazzi, E., Lanzani, C., Oneta, M., Parrilla, B., \& Persico, T. (2007). Safety of sperm washing and ART outcome in 741 HIV-1 sero-discordant couples. Hum Reprod; , 22, 772-777.

[68] Bujan, L., Hollander, L., Coudert, M., Gilling-Smith, C., Vucetich, A., Guibert, J., Vernazza, P., Ohl, J., Weigel, M., Englert, Y., Semprini, A. E., Th, C. R. E. A., \& network, E. (2007). Safety and efficacy of sperm washing in HIV-1-serodiscordant couples where the male is infected: results from the European CREAThE network. AIDS; , 21(14), 1909-14.

[69] Kashima, K., Takakuka, K., Suzuki, M., et al. (2009). Studies of assisted reproduction techniques (ART) for HIV-1 discordant couples using washed sperm and the nested PCR method: a comparison of the pregnancy rates in HIV-1 discordant couples and control couples. Jpn J Infec Dis.2009; , 62, 173-176.

[70] Ryder, R. W., Kamenga, C., Jinju, M., Mbuyi, N., Mbu, 1., \& Behets, F. (2005). Pregnancy and HIV-1 incidence in 178 married couples with discordant HIV-1 serostatus: additional experience at an HIV-1 counselling centre in the Democratic Republic of the Congo. Trop Med Int Health: , 482-487.

[71] Ezeanochie, M., Olagbuji, B., Ande, A., \& Oboro, V. (2009). Fertility preferences, condom use and concerns among HIV-positive women in serodiscordant relationships in the era of antiretroviral therapy. Int J GynaecolObstet; , 10, 97-98.

[72] Kambin, S., \& Batzer, F. (2004). Assisted reproductive technology in HIV serodiscordant couples. Sexuality, Reproduction and Menopause.2004; , 2, 92-100.

[73] Gilling-Smith, C., Nicopoullos, Semprini. A. E., Frodsham, L. C., (2006, , \& , H. I. (2006). HIV and reproductive care-a review of current practice; , 113, 869-878.

[74] Englert, Y., Lesage, B., Van Vooren, J. P., et al. (2004). Medically assisted reproduction in the presence of chronic viral disease. Hum Reprod Update; , 10, 149-162. 